\title{
REVIEW
}

\section{Guidelines for musculoskeletal ultrasound in rheumatology}

\section{Department of Rheumatology and Clinical Immunology, Charité University Hospital, Humboldt University, Berlin, Germany \\ M Backhaus \\ G-R Burmester}

Department of Rheumatology and Physical Medicine, University Hospital, Zurich, Switzerland T Gerber

Department of Rheumatology, University of Ancona, Jesi, Italy W Grassi

Division of Rheumatology, Department of Internal Medicine III, University of Vienna, Austria

K P Machold

Department of Rheumatology, Medisch Centrum Alkmaar, The Netherlands W A Swen

Department of Rheumatology, University of Leeds, United Kingdom R J Wakefield

Department of Internal Medicine III, Institute for Clinical Immunology and Rheumatology, University Erlangen, Germany

B Manger

Correspondence to: Dr B Manger, Department of Internal Medicine III, University Erlangen Krankenhausstr 12, D-91054 Erlangen, Germany

Accepted 19 March 2001

*The Working Group for Musculoskeletal Ultrasound in the EULAR Standing

Committee on International Clinical Studies including Therapeutic Trials

\author{
M Backhaus, G-R Burmester, T Gerber, W Grassi, K P Machold, W A Swen, \\ R J Wakefield, B Manger $\star$
}

\section{Introduction}

Within the past decade, musculoskeletal ultrasound (US) has become an established imaging technique for the diagnosis and follow up of patients with rheumatic diseases. ${ }^{1-5}$ This has been made possible through technological improvements, resulting in faster computers and higher frequency transducers. US is most commonly used in the assessment of soft tissue disease or detection of fluid collection and can also be used to visualise other structures, such as cartilage and bone surfaces. ${ }^{67}$ Owing to the better axial and lateral resolution of US, even minute bone surface abnormalities may be depicted. Thus destructive and/or reparative/ hypertrophic changes on the bone surface may be seen before they are apparent on plain $x$ rays or even magnetic resonance imaging. ${ }^{8}$ However, US wave frequencies cannot penetrate into bone, therefore imaging of intra-articular disease is usually not possible. The "real time" capability of US allows dynamic assessment of joint and tendon movements, which can often aid the detection of structural abnormalities. Advantages of US include its non-invasiveness, portability, relative inexpensiveness, lack of ionising radiation, and its ability to be repeated as often as necessary, making it particularly useful for the monitoring of treatment. US can also be used for guidance of aspiration, biopsy, and injection treatment. ${ }^{9}$ Most musculoskeletal work is performed using "grey scale", which means images are produced in a black and white format; each white dot in the image represents a reflected sound wave. Sound waves travel in a similar way to light waves and therefore the denser a material is-for example, bone cortex, the more reflective it is and the whiter it appears on the screen. Water is the least reflective body material and therefore appears as black as the sound waves travel straight through it.

Newer US techniques, which are currently being evaluated, include colour and power Doppler imaging, which provide colour maps of tissues. Here the amount of colour is related to the degree of blood flow, which may be of use in assessment of vascular tissues as may occur in soft tissue inflammation. ${ }^{10}$ To increase further the sensitivity of power Doppler intravenous bubble contrast agents are under development.
There are few data about which imaging modality is most appropriate in any given situation. Only rarely have the diagnostic values of different imaging techniques in various conditions been compared. ${ }^{11-13}$ As US is evolving, its place in patient management is becoming increasingly clear.

\section{Technical equipment}

High quality, high resolution equipment is essential for musculoskeletal work. The choice of transducer will depend on the type of examinations likely to be undertaken. High frequency $(7.5-20 \mathrm{MHz})$, linear transducers are generally best for demonstrating superficial structures such as tendons, ligaments, and small joints, whereas low frequency transducers (3.5-5 $\mathrm{MHz}$ ) are sometimes more suited for larger or deeper sited joints such as the shoulder or hip. ${ }^{44}$ In US there is a constant compromise between image resolution and depth of penetration of the sound waves. Higher frequency transducers provide better spatial resolution, but these transducers have a shallower depth of penetrance than a lower frequency transducer. The size of the footprint (the surface area of the transducer in contact with the skin) is also an important factor in examination technique. For example, transducers with a large footprint are often inadequate to visualise fully small joints such as the metacarpophalangeal joints as they cannot be manoeuvred adequately. However, these are only general considerations; the critical issue is the overall image resolution, which has to be analysed and compared carefully before a purchase is made. For practical reasons it is recommended to test whether with a particular piece of sonographic equipment the fine definition of small structures can be seen, such as the insertion of a small extensor tendon of the finger or the tiny quantity of fluid normally detectable in the pre-Achilles bursa.

The practical value of colour Doppler/power Doppler capabilities is still under investigation, especially considering the additional cost. The rationale for colour/power Doppler is the detection of increased soft tissue perfusion. The potential application of three dimensional US is also currently under evaluation. Finally, consideration needs to be given to methods of 
Table 1 Transducer orientation in standardised musculoskeletal examination

\begin{tabular}{lll}
\hline & Longitudinal scan & Transverse scan \\
\hline $\begin{array}{l}\text { Left side of the screen } \\
\text { Right side of the screen }\end{array}$ & Proximal, cranial, upper & Medial, ulnar, tibial \\
& Distal, caudal, lower & Lateral, radial, fibular \\
\hline
\end{tabular}

image documentation. In general, every examination should be carefully documented. Images may be recorded on paper, films, video cassettes, laser printed ' $x$ ray' acetates, optical discs, and digital storage systems. All demonstrated structures should be documented in a standardised way to ensure a better reproducibility of these results. Pathological findings should be documented in two perpendicular planes. Table 1 gives the transducer orientation.

\section{Teaching and training}

As US is the most operator dependent imaging modality, the experience and expertise of the examiner will determine the value of the diagnostic information obtained. Knowledge about the basic principles relevant to sound waves and a detailed knowledge of anatomy is mandatory. Although the procedure itself has no specific side effects, harm may result from incorrect acquisition and interpretation of images owing to operator inexperience. To standardise the quality of musculoskeletal US education, national and international societies - for example, EULAR, have established training guidelines for US. Training courses have been organised by experts in US providing "hands on" experience. However, musculoskeletal US cannot be learnt at a conference over a few days. There is no substitute for proper training under the guidance of an experienced investigator. Continuous training and education of people performing US is essential. Beginners are therefore encouraged to seek local expertise, where it is available, and the authors of this article are glad to be of help in establishing such contacts in their respective countries.

US is most valuable in a clinical setting, in which the clinician can interpret the images in the light of the clinical history and physical examination, enabling ultrasound to become the physician's extended finger. ${ }^{4}{ }^{15}$ However, in some instances, especially for a scientific analysis of the relative values of various imaging modalities, a second view point of an experienced sonographer is necessary to balance the possibility of "seeing" what one expects to see already from a clinical examination.

4. Standardisation of musculoskeletal US In the following paragraphs a list of detectable diseases, patient positioning, and standard scans are given. Representative images are provided for a selection of scans. An extensive version with a complete collection of all images of standard scans given can be seen on the internet at the official EULAR web site (www.eular.org). A link at the bottom of the right side of the screen leads to "Imaging in Rheumatology" (www.sameint.it/eular) and from there to the "Working Group for Musculoskeletal Ultrasound in Rheumatology".
4.1. SHOULDER JOINT

The polyarticular manifestation of rheumatic diseases frequently leads to symptoms earlier in weightbearing joints of the leg. Arthritic joints of the arm may exhibit relatively few symptoms despite marked inflammation. An early detection of changes of tendons, bursae, rotator cuff, and cartilage is possible by musculoskeletal US, which is essential to establish adequate treatment. To detect inflammatory lesions the anterior, lateral, and posterior, longitudinal and transverse scans with rotation of the shoulder are most helpful. A sensitive technique for finding even very small shoulder effusions is the axillary longitudinal scan, but elevation of the arm may not be possible for patients with advanced disease.

\subsubsection{US detectable pathology}

1 Rotator cuff:

- Tear (complete/ partial)

- Calcific tendinitis

2 Biceps tendon:

- Tear (complete/partial)

- Dislocation

- Tenosynovitis

- Effusion in the bicipital groove

3 Subcoracoid/subacromial/subdeltoid bursa:

- Bursitis

4. Axillary recessus:

- Synovial proliferation

- Effusion

5 Humeral head:

- Irregular contour

- Bone and cartilage lesions (erosions, osteophytes, Hill-Sachs lesion)

6 Joint space:

- Loose joint bodies

- Osteochondromatosis

7 Acromioclavicular joint:

- Dislocation

- Synovial proliferation/effusion

- Irregular bone profile

8 Deltoid muscle

- Haematoma

- Tear

4.1.2. Positioning of the patient

- Sitting position

- $90^{\circ}$ flexion of the elbow joint

- The hand should be positioned in supination on top of the patient's thigh

- For a dynamic examination, active and/or passive external and internal rotation of the humerus over the full range of motion with $90^{\circ}$ flexed elbow is recommended

\subsubsection{Standard scans}

1 Anterior transverse scan in neutral position (fig 1)

2 Anterior transverse scan in maximal internal rotation (fig 2)

3 Anterior longitudinal scan

4 Anterior longitudinal scan in maximal internal rotation

5 Lateral longitudinal scan in neutral position

6 Lateral longitudinal scan in maximal internal rotation

7 Posterior transverse scan

8 Axillary longitudinal scan with raised arm

9 Acromioclavicular joint scan 

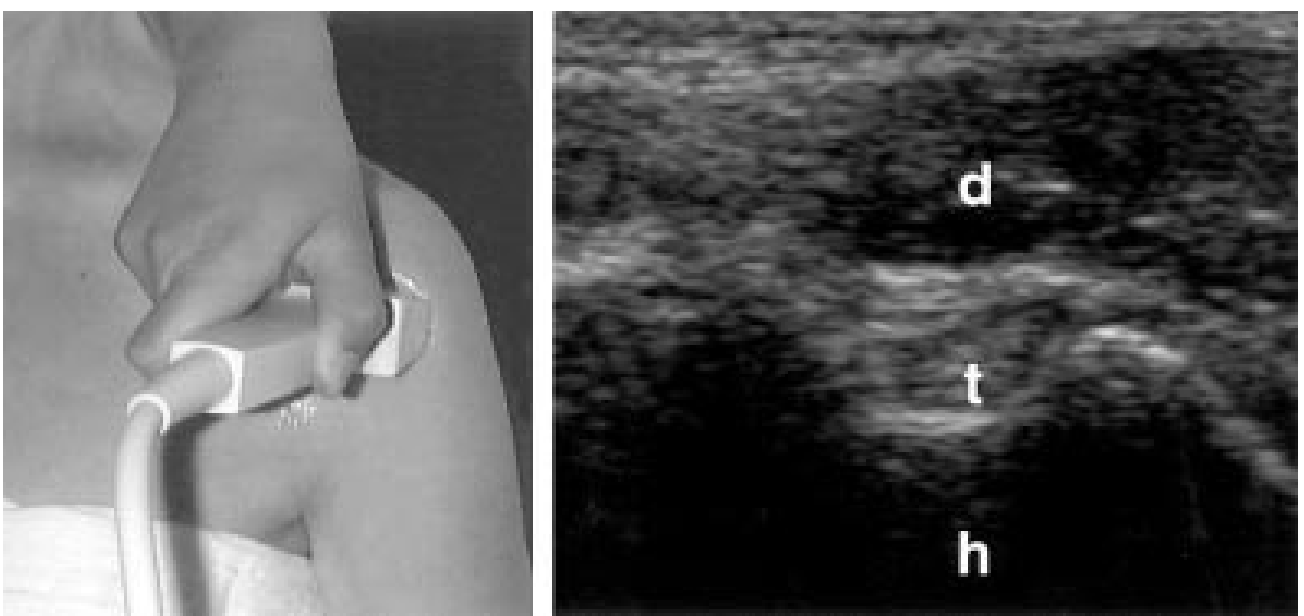

Figure 1 Anterior transverse scan in neutral position at the bicipital groove. $h=$ humerus; $t=$ biceps tendon; $d=$ deltoid muscle.

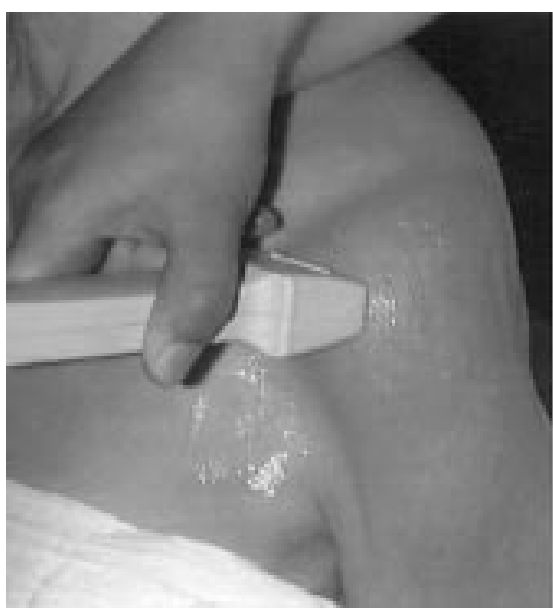

4.2. ELBOW JOINT

Inflammatory lesions in the elbow can most easily be detected early in the disease process in ventral longitudinal scans over the humeroradial and humeroulnar joints. Another common location of synovitis is the olecranon fossa.

\subsubsection{US detectable pathology}

1 Humeroradial joint:

- Synovial proliferation

- Effusion

- Bony lesion

- Loose joint body

2 Humeroulnar joint:

- Synovial proliferation

- Effusion

- Bony lesion

- Loose joint body

3 Olecranon fossa:

- Synovial proliferation

- Effusion

4 Olecranon bursa:

- Bursitis

5 Lateral/medial humeral epicondylus:

- Epicondylitis (lateral and medial)

6 Ulnar nerve:

- Compression

- Morphostructural changes

7 Subcutaneous tissue:

- Rheumatoid nodule

- Tophi

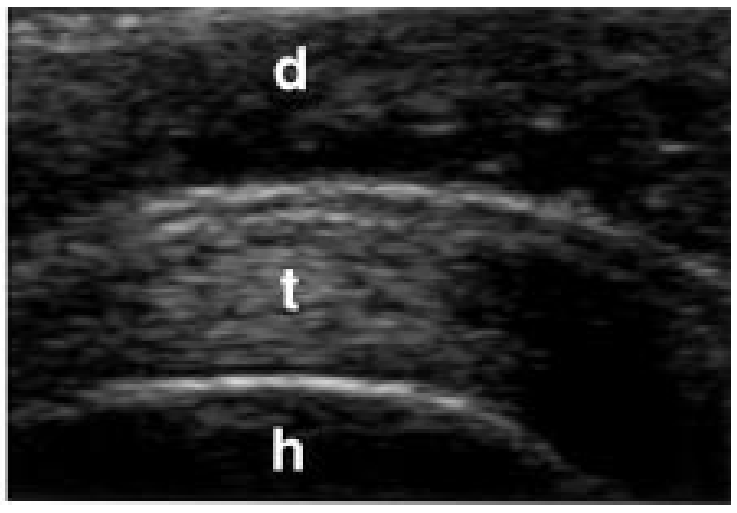

Figure 2 Anterior transverse scan in maximal internal rotation of the shoulder. $h=$ humerus; $t=$ supraspinatus tendon; $d=$ deltoid

\subsubsection{Positioning of the patient}

- Sitting position

- Full extension of the elbow joint and supination of the lower arm (ventral scans)

- Flexion of the elbow joint in a $90^{\circ}$ angle (dorsal scans)

- For the dorsal scans the hand can be placed on the hip or on the thigh of the patient with moderate internal rotation of the humerus

\subsubsection{Standard scans}

1 Anterior humeroradial longitudinal scan (fig 3)

2 Anterior humeroulnar longitudinal scan

3 Anterior transverse scan (fig 4)

4 Posterior longitudinal scan

5 Posterior transverse scan (fig 5)

6 Lateral longitudinal scan in extension

7 Lateral longitudinal scan in $90^{\circ}$ flexion

8 Medial longitudinal scan

\subsection{WRIST}

In many instances clinical examination of the wrist may be sufficient. With high frequency transducers of $10 \mathrm{MHz}$ and more, even minor synovitic lesions can be detected. US can also be helpful in differentiating synovial and tenosynovial pathology and examining morphostructural changes of the median nerve in carpal tunnel syndrome. 


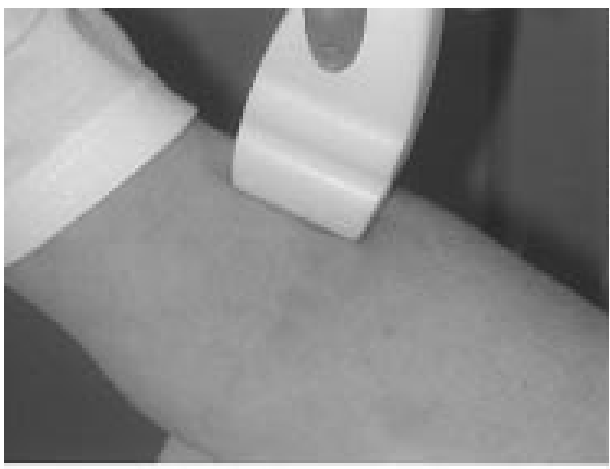

Figure 3 Anterior humeroradial longitudinal scan at the elbow. $h=$ humerus; $r=$ radius; $m=$ muscles $;^{\circ}=$ articular cartilage

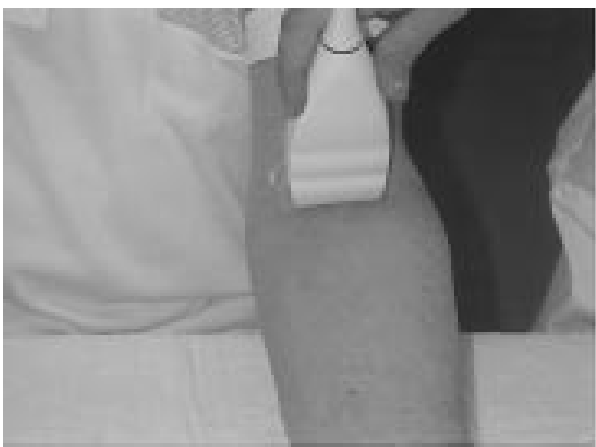

Figure 4 Anterior transverse scan at the distal humeral epiphysis. $h=$ humerus $;^{\circ}=$ articular cartilage; $m=$ muscles.

4.3.1. US detectable pathology

1 Carpal tunnel:

- Tenosynovitis

- Morphostructural changes of median nerve

- Ganglion

2 Extensor tendons:

- Tenosynovitis

- Alterations of extensor tendons

- Rheumatoid nodules

- Ganglion

3 Radio-ulno-carpal joint:

- Synovial proliferation

- Effusion

- Ganglion

- Lesions of triangular fibrocartilage complex

- Calcification

- Bony lesions (erosions, osteophytes)

4.3.2. Positioning of the patient

- Sitting position

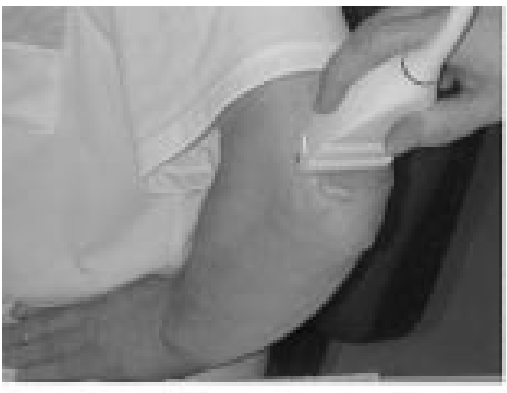

Figure 5 Posterior transverse scan at the distal humeral epiphysis. $h=$ humerus $;^{\circ}=$ articular cartilage; $m=$ triceps muscle.
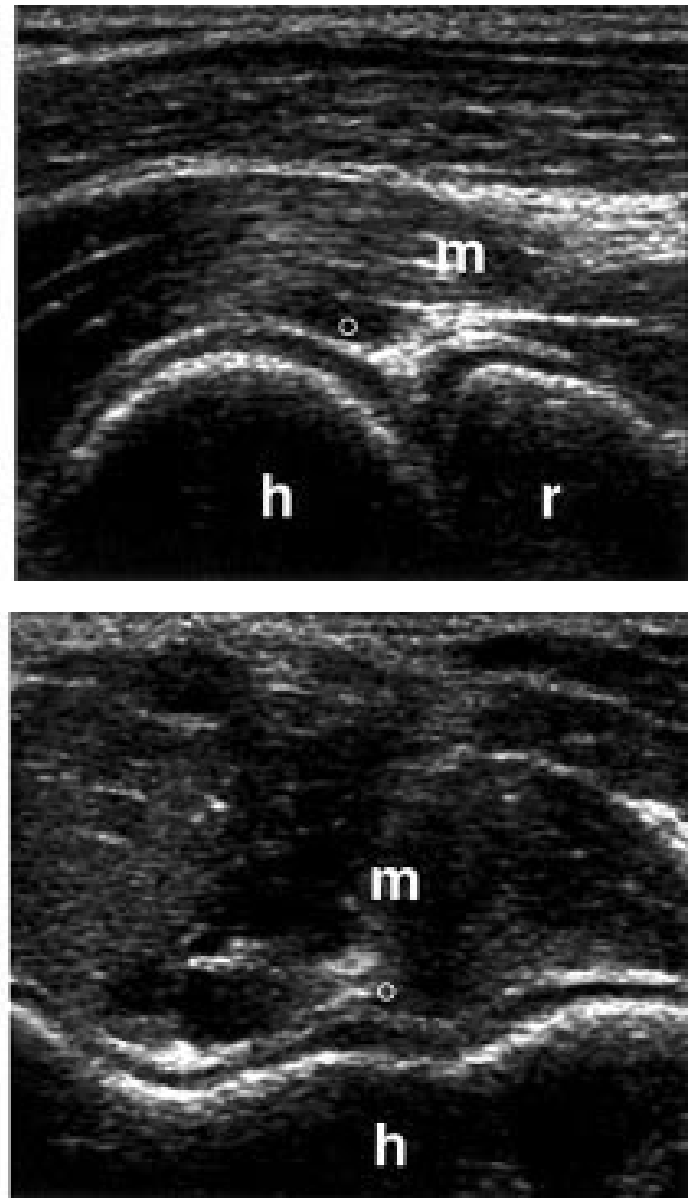

- Positioning of the hand on top of the thigh or on an examining table

- Dynamic examination with active flexion/ extension of the fingers

\subsubsection{Standard scans}

1 Volar transverse scan (fig 6)

2 Volar longitudinal scan

3 Dorsal transverse scan (radial)

4 Dorsal transverse scan (ulnar)

5 Dorsal longitudinal scan (radial)

6 Dorsal longitudinal scan (median)

7 Dorsal longitudinal scan (ulnar)

\subsection{HAND}

Finger joints are easily accessible to clinical examination. With high frequency transducers of $10 \mathrm{MHz}$ and more, even minor synovitic lesions can be detected. US can also be helpful

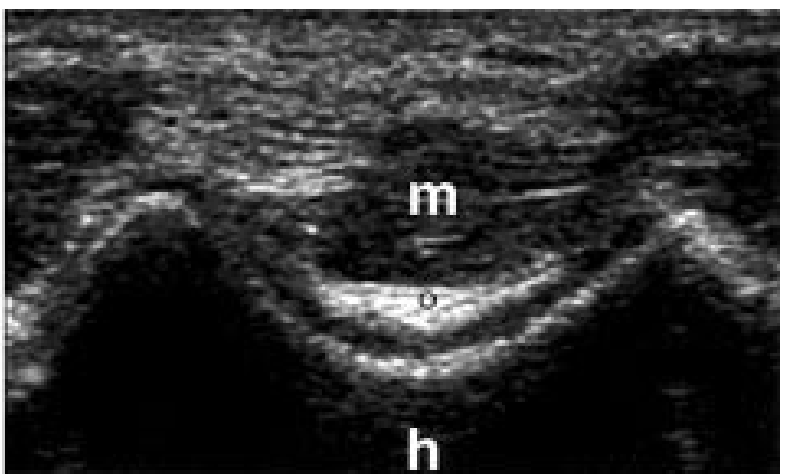



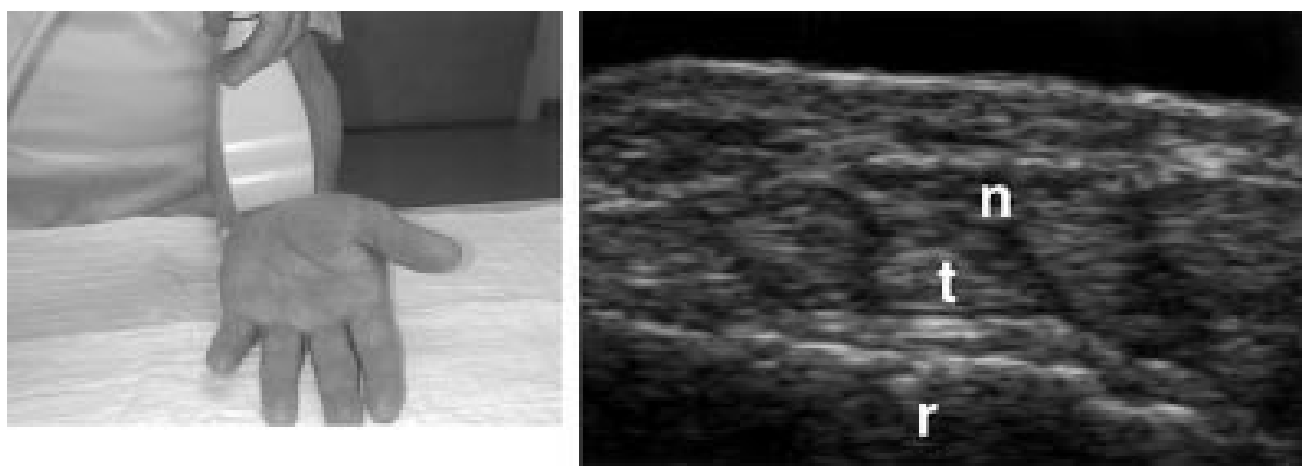

Figure 6 Volar transverse scan at the carpal tunnel. $r=$ radius; $n=$ median nerve; $t=$ flexor tendons

in differentiating synovial and tenosynovial pathology.

\subsubsection{US detectable pathology}

1 Effusion/synovial proliferation

2 Synovial cysts

3 Tendinitis/tenosynovitis/tendon tear

4 Cartilage thinning/lesion

5 Bony lesion (erosion, change of the bone profile, osteophyte)

6 Articular dislocation

7 Ganglion

8 Periarticular lesions: rheumatoid nodules, crystal deposition, calcinosis

4.4.2. Positioning of the patient

- See wrist joint (4.3.2.)

\subsubsection{Standard scans}

1 Dorsal longitudinal scan

2 Dorsal transverse scan (fig 7)

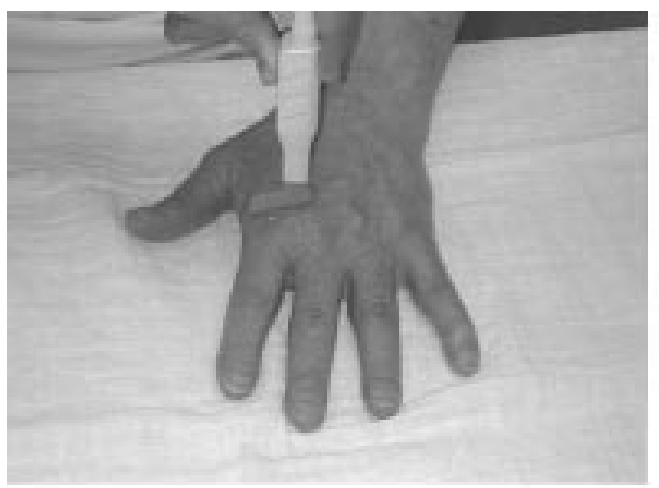

Figure 7 Dorsal transverse scan at the metacarpal head. $m h=$ metacarpal head; $t=$ extensor tendon.
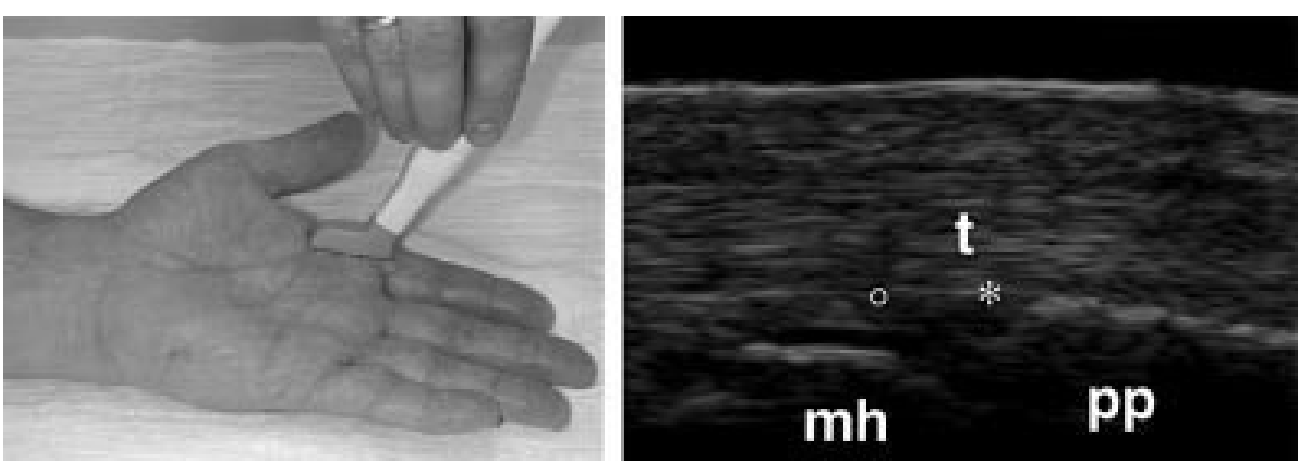

Figure 8 Palmar longitudinal scan at the metacarpophalangeal joint. ${ }^{*}=$ joint cavity; ${ }^{\circ}=$ articular cartilage; $p p=$ proximal phalanx; $m h=$ metacarpal head; $t=$ flexor tendon .

3 Palmar longitudinal scan (figs 8 and 9)

4 Palmar transverse scan (fig 10)

5 Thenar longitudinal scan

6 Thenar transverse scan

7 Hypothenar longitudinal scan

8 Hypothenar transverse scan

9 Lateral longitudinal scan (proximal interphalangeal (PIP) and metacarpophalangeal I, II, V joints)

10 Medial longitudinal scan (PIP joints)

4.5. HIP

Only rarely can effusions of the hip joint be detected by clinical examination. Here US is most helpful to detect effusion and synovitis especially before arthrocentesis. The anterior longitudinal scan parallel to the femoral neck is most valuable for the detection of an effusion as well as erosions or osteophytes. The anterior transverse scan after $90^{\circ}$ rotation is necessary

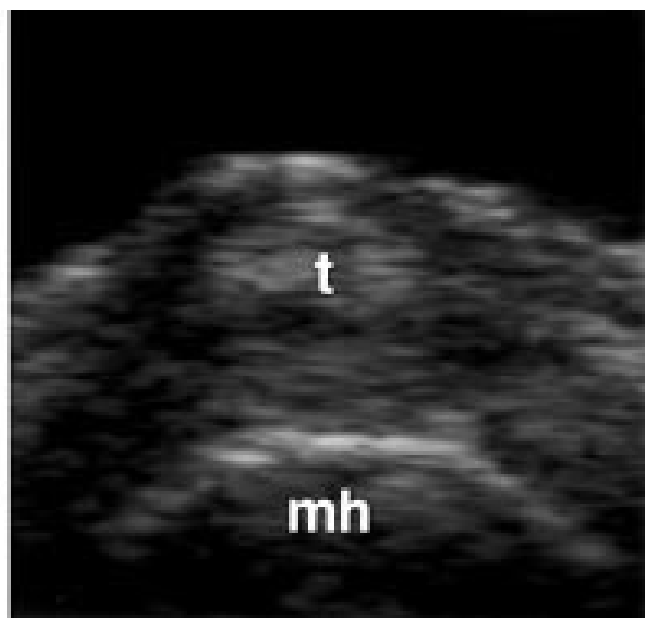



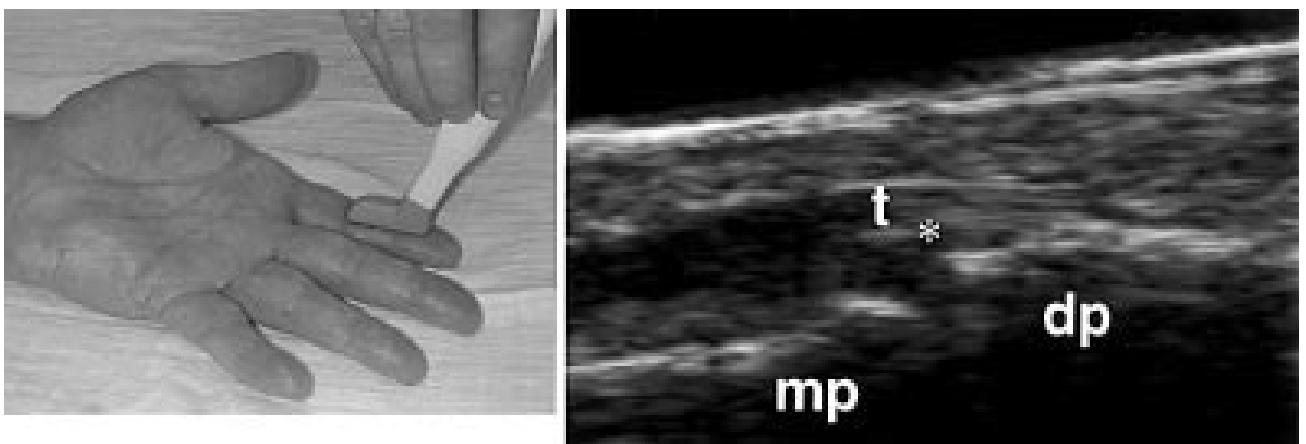

Figure 9 Palmar longitudinal scan at the distal interphalangeal joint. ${ }^{*}=$ joint cavity; $d p=$ proximal phalanx; $m p=$ middle phalanx; $t=$ flexor tendon.

before arthrocentesis to define the optimal location and identify vessels which should be avoided. The lateral longitudinal scan is helpful to detect a trochanteric bursitis.

4.5.1. US detectable pathology

1 Joint effusion/synovial proliferation

2 Cartilage lesion

3 Bony lesion (erosion, osteophyte, irregular bone surface, slipped capital femoral epiphysis)

4 (Osteo-)chondromatosis

5 Loose joint body

6 Bursa trochanteric/iliopectineal bursitis

7 Infection or loosening of prosthesis

8 Calcifications

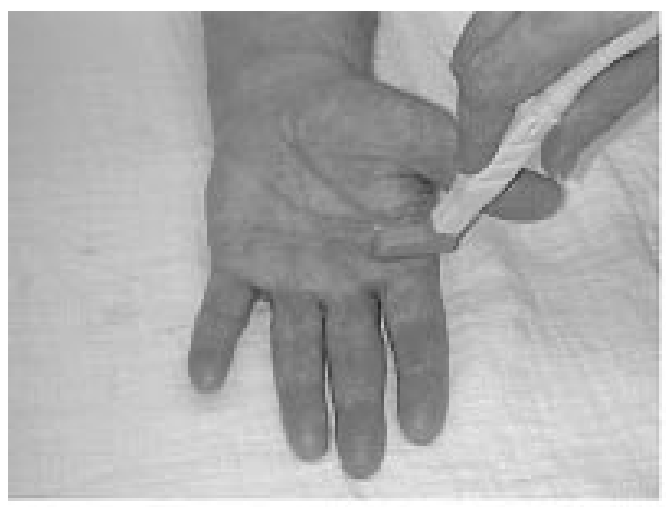

Figure 10 Palmar transverse scan at the metacarpal head. $m h=$ metacarpal head $;^{\circ}=$ articular cartilage $t=$ flexor tendon.

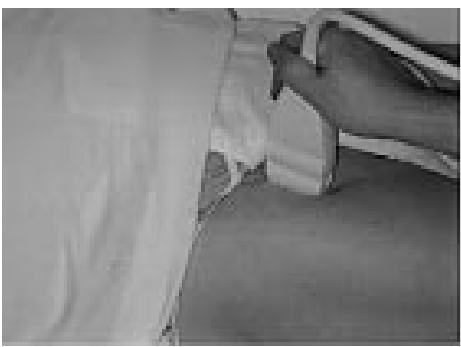

Figure 11 Anterior longitudinal scan at the hip. $a=$ acetabulum; $f=$ femur; ${ }^{\star}=$ joint cavity; $m=$ muscles.
4.5.2. Positioning of the patient

- Supine position

- Hip joint in neutral position

4.5.3. Standard scans

1 Anterior longitudinal scan (fig 11)

2 Anterior transverse scan

3 Lateral longitudinal scan

\subsection{KNEE}

In contrast with the hip, the knee joint is easily accessible to clinical examination. However, very small effusions or synovitic proliferations which are missed clinically can often be demonstrated by US. Small amounts of effusion can be detected in the suprapatellar
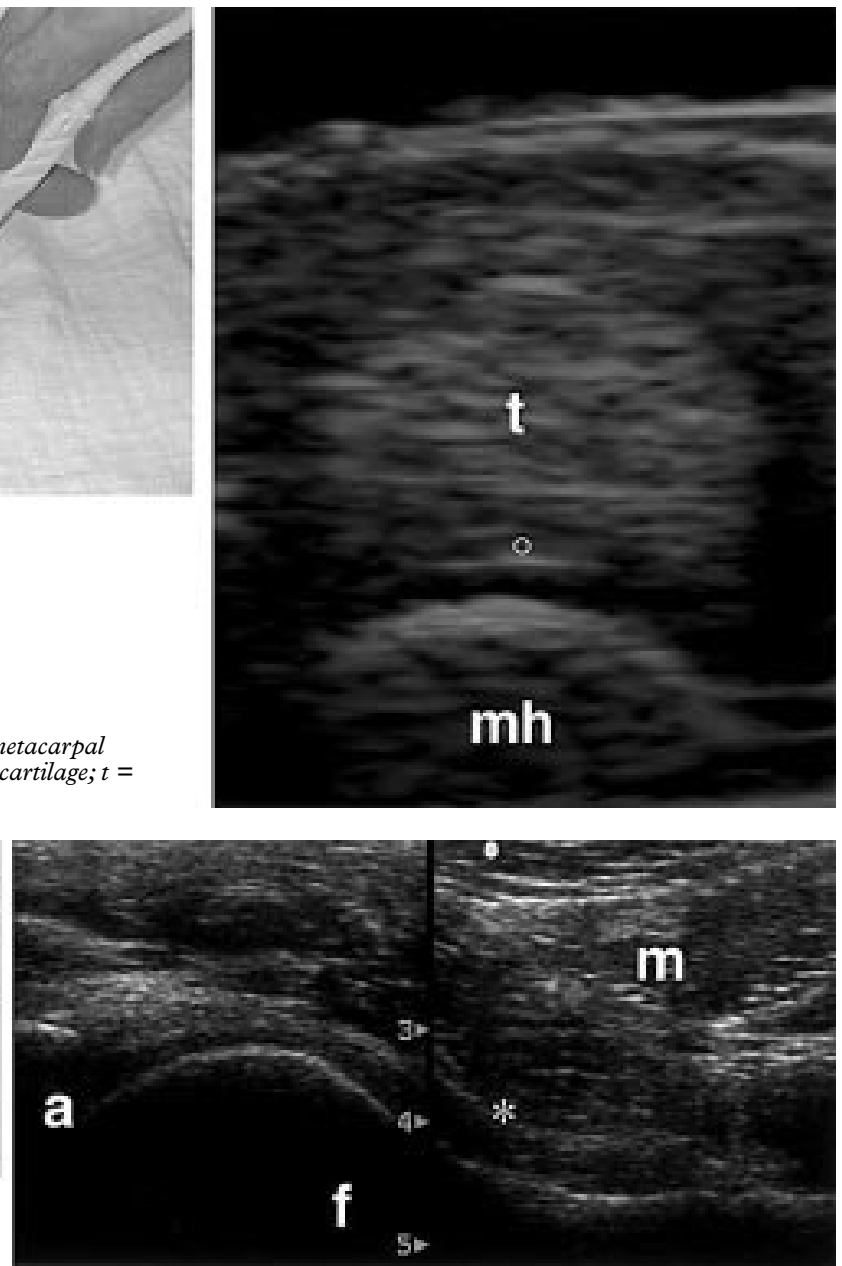

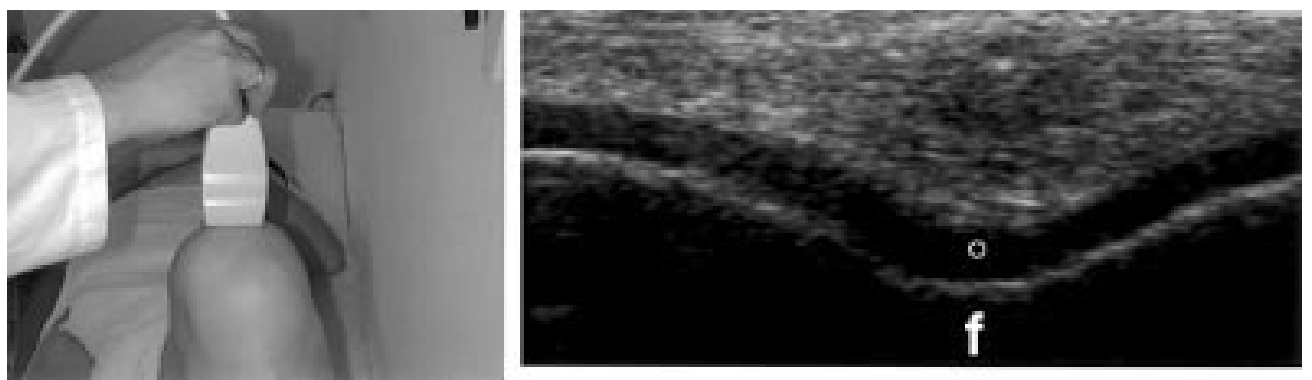

Figure 12 Suprapatellar transverse scan in maximal flexion. $f=$ femur $;^{\circ}=$ articular cartilage.

longitudinal and transverse scans in neutral position when pressure is exerted on the suprapatellar and parapatellar pouch by tightening of the quadriceps muscle.

An important indication for musculoskeletal US is the examination of pathological processes of the popliteal region. Popliteal cysts (Baker's cysts) are fluid accumulation in the bursa of the gastrocnemius or semimembranosus muscles. Frequently those cysts communicate with the joint space. To confirm the diagnosis of a popliteal cyst this comma shaped extension has to be visualised sonographically in the posterior transverse scan between the medial head of gastrocnemius and semimembranosus tendon. Popliteal cysts can extend far into thigh and calf muscles and US allows precise definition of their shape and size. A rupture of a popliteal cyst, which may clinically mimic a deep vein thrombosis, is easily identified by US.

Loose joint bodies in the knee can be detected sonographically in the suprapatellar pouch and in the infrapatellar and popliteal regions. However, the failure to detect a loose body in the knee or any other joint can never rule out its presence.

\subsubsection{US detectable pathology}

1 Suprapatellar and parapatellar pouch:

- Synovial proliferation

- Synovial folds

- Effusion

2 Quadriceps tendon:

- Tear (partial or complete)

3 Femoropatellar joint:

- Irregular contours

- Bony lesions (erosions, osteophytes)

4 Popliteal sulcus:

- Bursitis

- Synovial proliferation

5 Patellar ligament:

- Tear (partial/complete)

6 Deep infrapatellar bursa:

- Bursitis

7 Subcutaneous prepatellar bursa:

- Bursitis

8 Tuberosity of tibia:

- Irregular bony contour (Mb. OsgoodSchlatter)

- Infrapatellar bursitis

9 Ligaments:

- Tear/lesion

10 Meniscus (lateral/medial):

- Lesion

- Cyst

11 Popliteal fossa:
- Popliteal cyst (volume, echogenicity signs of leakage)

- Compression of vessels

4.6.2. Positioning of the patient

- Supine position for ventral and lateral scans

- Prone position for dorsal scans

- Knee joint in neutral position and/or $30^{\circ}$ flexion

- Maximal flexion for imaging of the intercondylar sulcus

- Dynamic examination of the suprapatellar pouch with relaxed and contracted quadriceps muscle

\subsubsection{Standard scans}

1 Suprapatellar longitudinal scan

2 Suprapatellar transverse scan in neutral position

3 Suprapatellar transverse scan in maximal flexion (fig 12)

4 Infrapatellar longitudinal scan

5 Infrapatellar transverse scan

6 Medial longitudinal scan

7 Lateral longitudinal scan

8 Posterior medial longitudinal scan

9 Posterior lateral longitudinal scan

10 Posterior transverse scan

\subsection{ANKLE AND HEEL}

Inflammatory changes of the ankle and talocalcaneonavicular joints are easily detectable by US, as are tenosynovitis of tibialis anterior, posterior, and peroneus tendons.

The Achilles tendon can by examined by US in its full length, and calcifications, ruptures, and bursitis can be differentiated. In patients with heel pain, lesions of the plantar fascia, calcaneus spurs, and erosions can be detected sonographically.

\subsubsection{US detectable pathology}

1 Ankle and talocalcaneonavicular joint:

- Synovial proliferation

- Effusion

- Cartilage lesions

- Bony lesions

- Loose joint body

- (Osteo-)chondromatosis

2 Tibial anterior muscle:

- Tenosynovitis/tear

3 Tibial posterior muscle:

- Tenosynovitis/tear

4 Peroneus long./brev. muscles:

- Tenosynovitis/tear

5 Achilles tendon:

- Calcification 


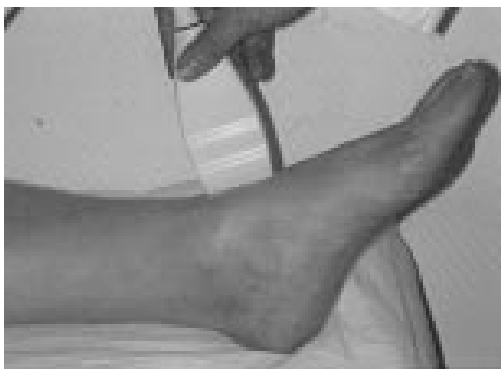

Figure 13 Anterior longitudinal scan at the ankle. tib $=$ tibia; tal $=$ talus $;^{\circ}=$ articular cartilage.
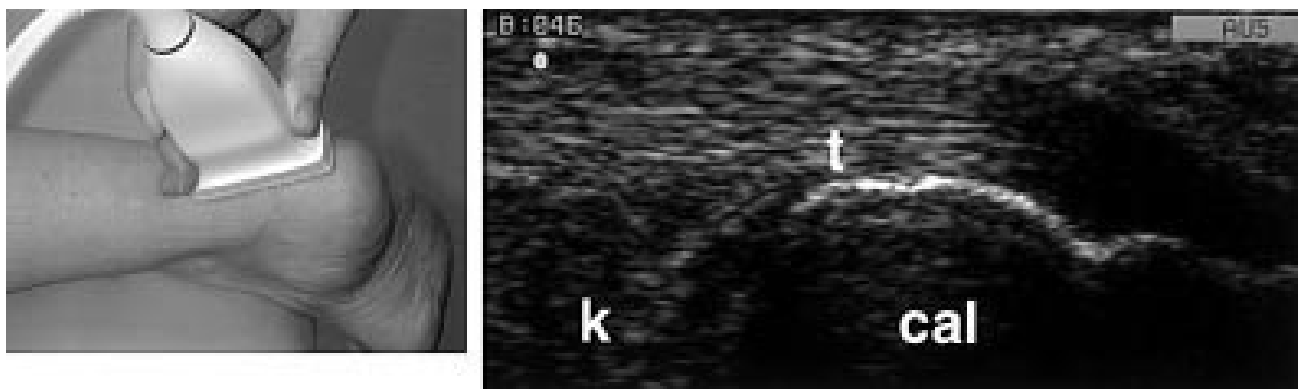

Figure 14 Posterior longitudinal scan at the heel. $t=$ achilles tendon; cal $=$ calcaneus; $k=$ Kager's fat pat.
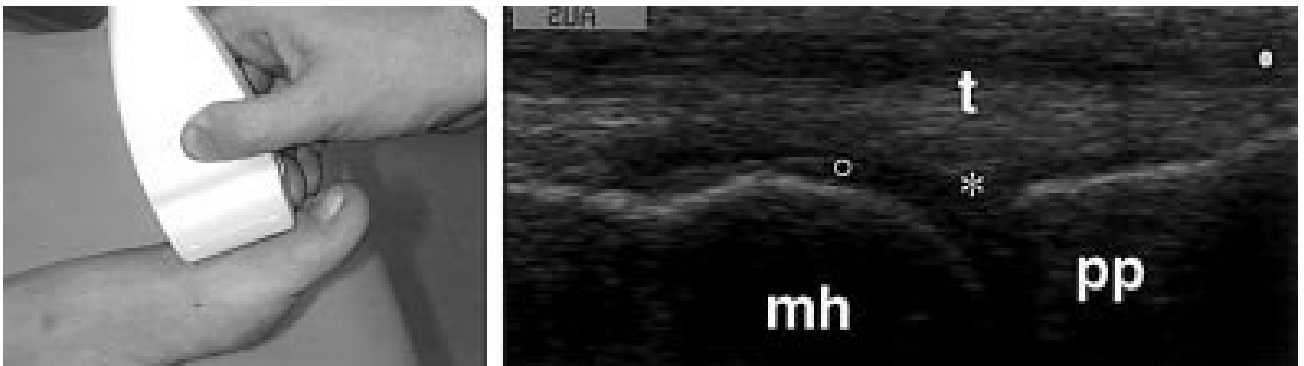

Figure 15 Dorsal longitudinal scan at the first toe. $m h=$ metatarsal head; $; p p=$ proximal phalanx; $t=$ extensor tendon; $\star=$ joint cavity $;^{\circ}=$ articular cartilage.

- Tear (partial/complete)

- Tendinitis/paratendinitis

- Nodules (tophy, rheumatoid nodules, xanthomas)

- Bursitis (retrocalcaneal or superficial)

6 Calcaneus:

- Calcification of plantar fascia

- Ossification (calcanaeus spur)

- Bony lesions (erosion)

4.7.2. Positioning of the patient

- Supine position for ventral and lateral scans

- Prone position for dorsal scan

- Hip and knee joints in neutral position

4.7.3. Standard scans

1 Anterior longitudinal scan (fig 13)

2 Anterior transverse scan

3 Perimalleolar medial longitudinal scan

4 Perimalleolar medial transverse scan

5 Perimalleolar lateral longitudinal scan

6 Perimalleolar lateral transverse scan

7 Posterior longitudinal scan (fig 14)

8 Posterior transverse scan

4.8. FOOT

Toes are easily accessible to clinical examination. With high frequency transducers of 10 $\mathrm{MHz}$ and more, even minor synovitic lesions can be detected. US can also be helpful in differentiating synovial and tenosynovial disease. Lesions of tophaceous gout can be identified by US because of their typical sound shadow.

\subsubsection{US detectable pathology}

1 Plantar fascia:

- Plantar fasciitis

2 Joints:

- Effusion/synovial proliferation

- Cartilage lesions

- Bone lesions (erosions, osteophytes)

3 Tendons:

- Tenosynovitis/tear

4 Subcutaneous tissue:

- Gout tophi

4.8.2. Positioning of the patient

- Supine position for the dorsal scans

- Prone position for plantar scans

\subsubsection{Standard scans}

All scans performed moving from proximal to distal.

1 Plantar longitudinal scan

2 Plantar transverse scan

3 Dorsal longitudinal scan (fig 15)

4 Dorsal transverse scan

5 Lateral scan (first and fifth toe) 
1 Gibbon WW, Wakefield RJ. Ultrasound in inflammatory disease. Radiol Clin North Am 1999;37:633-51.

2 Grassi W, Cervini C. Ultrasonography in rheumatology: an evolving technique. Ann Rheum Dis 1998;57:268-71.

3 Wakefield RJ, Gibbon WW, Emery P. The current status of ultrasonography in rheumatology. Rheumatology (Oxford) 1999;38:195-8.

4 Manger B, Kalden JR. Joint and connective tissue ultrasonography - a rheumatologic bedside procedure? A German experience. Arthritis Rheum 1995;38:736-42.

5 Manger B, Backhaus M. [Ultrasound diagnosis of rheumatic/inflammatory joint diseases.] Z Arztl Fortbild Qualitatssich 1997;91:341-5.

6 Grassi W, Tittarelli E, Pirani O, Avaltroni D, Cervini C. Ultrasound examination of metacarpophalangeal joints in rheumatoid arthritis. Scand J Rheumatol 1993;22:243-7.

7 Grassi W, Lamanna G, Farina A, Cervini C. Sonographic imaging of normal and osteoarthritic cartilage. Semin imaging of normal and osteoarth

8 Backhaus M, Kamradt T, Sandrock D, Loreck D, Fritz J, Wolf KJ, et al. Arthritis of the finger joints: a comprehensive approach comparing conventional radiography, scintigraapproach comparing conventional radiography, scintigraphy, ultrasound, and contrast-enhanced magnetic

9 Grassi W, Lamanna G, Farina A, Cervini C. Synovitis of small joints: sonographic guided diagnostic and therapeutic approach. Ann Rheum Dis 1999;58:595-7.
10 Hau M, Schultz H, Tony HP, Keberle M, Jahns R, Haerten $\mathrm{R}$, et al. Evaluation of pannus and vascularization of the metacarpophalangeal and proximal interphalangeal joints in rheumatoid arthritis by high-resolution ultrasound 2303-8.

11 Swen WA, Jacobs JW, Hubach PC, Klasens JH, Algra PR, Bijlsma JW. Comparison of sonography and magnetic resonance imaging for the diagnosis of partial tears of finger extensor tendons in rheumatoid arthritis. Rheumatology (Oxford) 2000;39:55-62.

12 Swen WA, Jacobs JW, Neve WC, Bal D, Bijlsma JW. Is sonography performed by the rheumatologist as useful as arthrography executed by the radiologist for the assessment of full thickness rotator cuff tears? J Rheumatol 1998;25: 1800-6.

13 Swen WA, Jacobs JW, Algra PR, Manoliu RA, Rijkmans J, Willems WJ, et al. Sonography and magnetic resonance imins Wu rotator cuff tears. Arthritis Rheum 1999;42:2231-8.

14 Grassi W, Tittarelli E, Blasetti P, Pirani O, Cervini C. Finger tendon involvement in rheumatoid arthritis. Evaluation with high-frequent in rheumatoid arthritis. Evaluation with high-fred

15 Leeb BF, Stenzel I, Czembirek H, Smolen JS. Diagnostic use of office-based ultrasound. Baker's cyst of the right knee joint. Arthritis Rheum 1995;38:859-61. 